\title{
Decoding Phase-Based Information from Steady-State Visual Evoked Potentials with Use of Complex-Valued Neural Network
}

\author{
Nikolay V. Manyakov, Nikolay Chumerin, Adrien Combaz, Arne Robben, \\ Marijn van Vliet, and Marc M. Van Hulle \\ Laboratory for Neuro- and Psychofysiology, K.U.Leuven, \\ Herestraat 49, bus 1021, 3000 Leuven, Belgium \\ \{NikolayV.Manyakov, Nikolay.Chumerin, Adrien.Combaz, Arne.Robben, \\ Marijn.vanVliet, Marc.VanHulle\} @med.kuleuven . be
}

\begin{abstract}
In this paper, we report on the decoding of phase-based information from steady-state visual evoked potential (SSVEP) recordings by means of a multilayer feedforward neural network based on multivalued neurons. Networks of this kind have inputs and outputs which are well fitted for the considered task. The dependency of the decoding accuracy w.r.t. the number of targets and the decoding window size is discussed. Comparing existing phase-based SSVEP decoding methods with the proposed approach, we show that the latter performs better for the larger amount of target classes and the sufficient size of decoding window. The necessity of the proper frequency selection for each subject is discussed.
\end{abstract}

\section{Introduction}

Research on brain-computer interface 1 (BCIs) has witnessed a tremendous development in recent years (see, for example, the editorial in IEEE Signal Processing Magazine [1]), and is now widely considered as a successful application of the neurosciences. BCIs can significantly improve the quality of life of patients suffering from amyotrophic lateral sclerosis, stroke, brain/spinal cord injury, cerebral palsy, muscular dystrophy, etc. Brain-computer interfaces are either invasive [2]3/4] or noninvasive [5/6]. The invasive ones use recordings made intracortically (local field potentials and action potentials) or from the surface of the brain (electrocorticogram), whereas the noninvasive ones mostly employ electroencephalograms (EEGs) recorded from the subject's scalp.

Several noninvasive methods have been proposed in the literature. The one we consider in this paper, is based on the steady-state visual evoked potential (SSVEP). This type of BCI relies on the psychophysiological properties of EEG

\footnotetext{
${ }^{1}$ A BCI is a device, which records and interprets brain activity automatically, allowing the subject to interact with the world through computers, robot actuators and so on, bypassing the need for muscular activity.
} 
brain responses recorded from the occipital area during the periodic presentation of visual stimuli (flickering stimuli). When the periodic presentation is at a sufficiently high rate $(>6 \mathrm{~Hz}$ ), the individual transient visual responses (which are time and phase locked to the stimulus onsets) overlap and become a steady state signal: the signal resonates at the stimulus rate and its multipliers 7 .

Conventional SSVEP-based BCI systems [8|9|10]11] use an increase in amplitude of frequencies $f, 2 f, 3 f, \ldots$ in the power spectral density of the EEG data to detect that the subject is looking at target flickering at rate $f$. Since the relevant EEG activity is always embedded into other on-going brain activity and contaminated by (recording) noise, the detection task is not straight-forward. Considering a small recording interval it is quite likely to detect an (irrelevant) increase in the amplitude at frequency $f$. To overcome this problem and improve the decoding performance, several methods are used: averaging over several time intervals [8, recording over longer period of time [9, preliminary training [10, etc. Finally, to enhance the BCI functionality, several stimulation frequencies $f_{1}, \ldots, f_{N}$ are used simultaneously at the same time, instead of only one $f$. In this case each frequency corresponds to a particular command with which the $\mathrm{BCI}$ can communicate. The detection problem, therefore, becomes more complex since now, the only one of several possible stimulation frequencies $f_{i}$ needs to be detected from the EEG recordings.

While these methods achieve good information transfer rate [11, their application using a computer screen (monitor) for visual stimulation has some limitations: the computer screen based stimulation is restricted by the refresh rate of the screen [12] (85 Hz in our case); only stimulations from some particular (and subject dependent) frequency interval produce good responses [10; the harmonics of some stimulation frequencies could coincide with the other frequencies (and their harmonics) deteriorating the decoding performance [12. These restrictions limit the number of target commands in SSVEP-based BCI. To be able to use more targets the differentiation between phases in SSVEP stimulation has been proposed in 13|14: even a single frequency could be used to encode commands employing the phase lag. For example, one can perform visual stimulation using $N$ targets simultaneously flickering with the same frequency $f$, but with different time delays $\Delta t_{m}=(m-1) /(f N)$ seconds for the command $m$ $(m=1, \ldots, N)$. Thus, extracting phase information from a Fourier transform of the EEG signal and comparing it to the phase of some reference signal(s) (for example, to the phase of EEG response for stimulus with zero phase lag [14] or to the phases of EEG responses for all possible delayed stimulations [13]), one can detect the target the subject is looking at. Such an approach allows to increase number of possible commands by combining different frequencies with different phase shifts [13] or simply using one (well detectable) frequency with different phase shifts [14. Here we account the problem of accurately detecting phase shifts using only short recording intervals, and properly assigning class labels to the estimated phase information. In this paper, we investigate the possibility of applying a multilayer neural network based on multi-valued neurons (MLMVN) [15] for decoding up to 16 phase shifted targets (vs. 4-6 in [13] and 8 
in [14), which uses information from any amount of harmonics (vs. one in 14]) and channels (vs. Oz in [14] and optimal or bipolar (Oz-POz) in [13]), and allow for a good approximation of circular data as we have in our case - the phase shifts and resulting classes are circular.

\section{Methods}

\subsection{EEG Experiment}

The EEG recordings have been performed with a prototype of a miniature wireless EEG system developed by imed and built around their ultra-low power 8-channel EEG amplifier chip [16. Each of the eight channels is recorded with sampling rate $f_{s}^{\mathrm{EEG}}=1000 \mathrm{~Hz}$ at resolution of $12 \mathrm{bits} / \mathrm{sample}$. We have used an electrode cap with large filling holes and sockets for active $\mathrm{Ag} / \mathrm{AgCl}$ electrodes (ActiCap, Brain Products). The eight electrodes were placed primarily on the occipital pole, namely at positions $\mathrm{Oz}, \mathrm{O} 1, \mathrm{O} 2, \mathrm{POz}, \mathrm{PO}$, PO3, PO4, PO8, according to the international 10-20 system. The reference and ground electrodes were placed on the left and right mastoids, respectively.

Four healthy subjects (all male, aged 24-34 with average age 29.5, three righthanded, one lefthanded) participated in the experiments. The experiment consisted of observing a flashing stimulus in the center of the screen (CRT monitor, refresh rate $f_{s}^{\text {scr }}=85 \mathrm{~Hz}$ ). To produce stronger SSVEP responses [12, we have used the (monitor) frame-based visual stimulation of period of six frames: three frames of intense (white) stimulus presentation followed by three frames without the stimulus (black). The stimulation frequency is, thus, $f=85 / 6 \approx$ $14.16 \mathrm{~Hz}$ close to $15 \mathrm{~Hz}$, which is reported to elicit the largest SSVEP amplitude 7 . The EEG data have been collected during sessions of two minutes long visual stimulation.

\subsection{Multilayer Feedforward Neural Network Based on Multi-valued Neurons}

Networks based on complex-valued neurons were reported to learn faster and generalize better than traditional neural networks in different benchmarks and real world problems $15[17] 18$. As was mentioned in [18, the use of complexvalued inputs/outputs, weights and activation functions make it possible to increase the functionality of a single neuron and of a neural network, to improve their performance and to reduce the training time.

We have used a multilayer feedforward neural network based on multi-valued neurons (MLMVN) [15|17]. Such a network incorporates derivative-free backpropagation training algorithms, resulting in fast convergence to the minimum of an error function [15]. In MLMVN, each neuron from every hidden or output layers has connections to all neurons from the previous layer and has a complex activation function $P(z)=z /|z|$, where $z=w_{0}+w_{1} x_{1}+\ldots+w_{n} x_{n}, x_{i} \in \mathbb{C}$ is

\footnotetext{
2 http://www.imec.be
} 
the output of the $i$-th neuron from previous layer (with $n$ neurons in this layer) and $w_{i} \in \mathbb{C}$ is the corresponding weight.

For the simulations we have used a MLMVN with 16 inputs, one hidden layer consisting of 24 neurons, and one neuron in the output layer. The network inputs are computed using phase information of the stimulation frequency and its first harmonic, estimated from all eight electrodes. Considering the EEG data window $\mathbf{E E G}(t)=\left(\operatorname{EEG}_{1}(t), \ldots, \operatorname{EEG}_{8}(t)\right)$ of length $T$ seconds $(t \in[0, T])$, the phase $\phi_{k, f}$ of the frequency $f$ from $k$-th channel $\mathrm{EEG}_{k}$ is estimated as:

$$
\phi_{k, f}=\arg \left(\int_{0}^{T} \operatorname{EEG}_{k}(t) \cos (2 \pi f t) d t+i \int_{0}^{T} \operatorname{EEG}_{k}(t) \sin (2 \pi f t) d t\right) .
$$

Since the phase information $\phi_{f, k} \in[0,2 \pi$ ) (where 0 and $2 \pi$ refer to the same phase), it can be represented by a complex number $e^{i \phi_{k, f}}$ on a unit circle on the complex plain. Estimated in such a way $16(8 \times 2)$ complex numbers constitute the input $\Phi=\left(e^{i \phi_{1, f}}, \ldots, e^{i \phi_{8, f}}, e^{i \phi_{1,2 f}}, \ldots, e^{i \phi_{8,2 f}}\right)$ to the MLMVN.

The output of the network $\mathcal{N}(\Phi)$ is such index $m(m=1, \ldots, N$, where $N$ is the number of target classes) of the unit circle sector, that $2 \pi(m-1) / N \leq$ $\arg P(z)<2 \pi m / N$. Thus, after the network is trained, classification is obtained by simply looking for the target class correspondent to the obtained unit circle sector.

\subsection{Training and Testing Data}

To validate the applicability and the accuracy of the proposed classifier, it is sufficient to limit ourselves to off-line decoding. For this assessment, we opted for a 4-fold cross-validation results. Namely, the recorded data have been split into four parts. One of those parts has been used for training and the rest for testing. All possible selections of data for training were done, leading to four decoding results. Since we recorded data from one flickering stimuli having no phase shifts, we introduced phase-shifted targets (stimuli) artificially in the next way. Considering that under the normal conditions individual SSVEP latency is stable 1319, the delay in the stimulation (using the same frequency) would introduce the same delay in the recorded EEG data. In this way, using proper shifts in the EEG data, the EEG responses for different phase shifted stimulations (with fixed frequency $f$ ) are constructed. From those constructed EEGs, we cut a number of intervals with length $T$ second in such a way, that starting points of those intervals are aligned to the stimulus onsets. As a consequence, the interval starting points are distanced between each other on integer number of periods (with the length $1 / f \mathrm{~s}$ ). We have used this strategy to generate the training and test data. While in the training set, we allowed the constructed data to be highly overlapped (to have bigger amount of training samples), we tried to reduce those overlaps in the test set?

\footnotetext{
${ }^{3}$ As we found later on, such introduced sparsity does not much influence the decoding accuracy.
} 


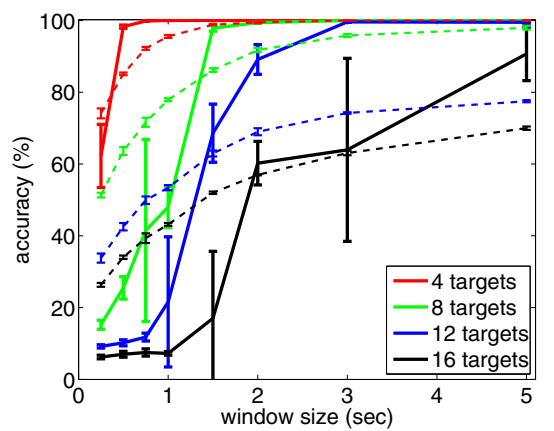

(a)

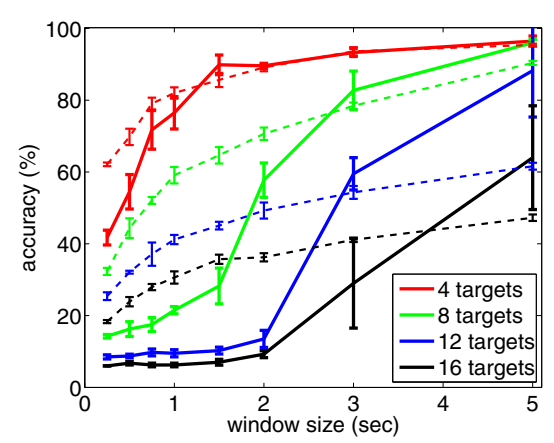

(c)

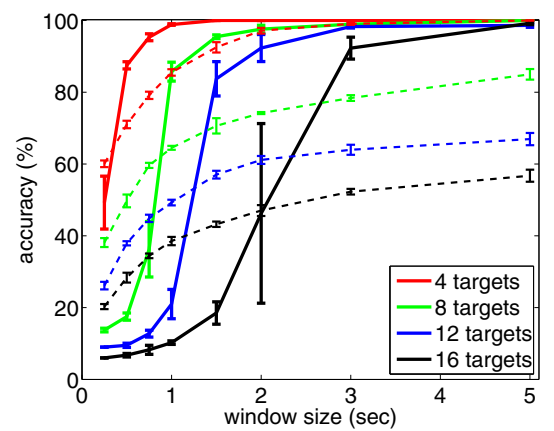

(b)

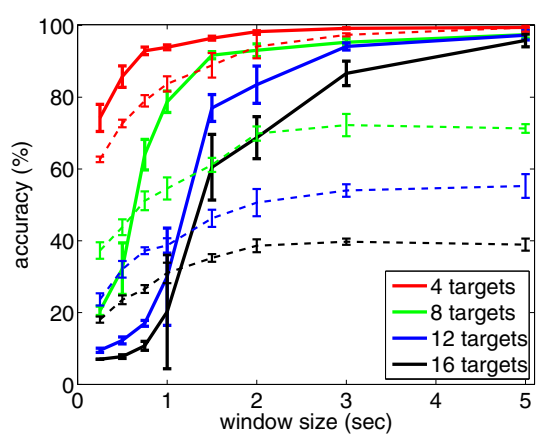

(d)

Fig. 1. Decoding results for 4 subjects based on the MLMVN (solid lines) and an extended version of the method from 13 (dashed lines). The results are shown for 4 , 8,12 , and 16 targets (phase shifts). Stimulation frequency is $f=85 / 6 \mathrm{~Hz}$. Error bars show standard deviations.

\section{Results and Discussion}

The results for four subject with stimulation $f=85 / 6 \mathrm{~Hz}$ and different number $N$ of target classes are shown in Fig. 1. together with the averaged among all subject performance in Fig. 2a,

As it can be seen from those figures, MLMVN gives good classification performance for all subjects for a proper window length. But this accuracy strongly depends on the number of targets: good performance is achieved starting from $T=1-1.5$ second window size for four targets, $2-3$ second window for eight targets, above 3 second window for 12 targets and so on.

As it can be seen from Fig. 1c, one subject performance is quite low compared to the other subjects. This could be due to the fact, that this subject does not generate a proper SSVEP with the chosen frequency $(85 / 6 \mathrm{~Hz})$. To validate this, we made a second test with this subject, but with the lower frequency of 


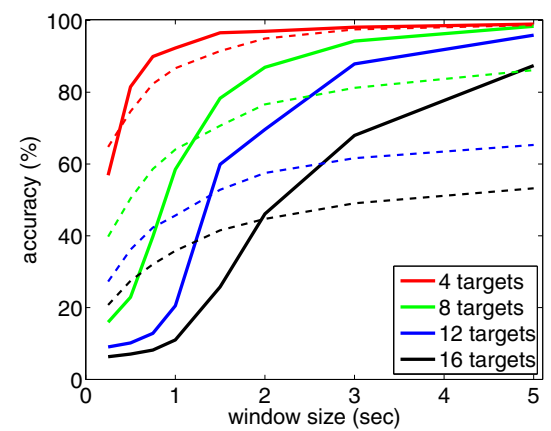

(a)

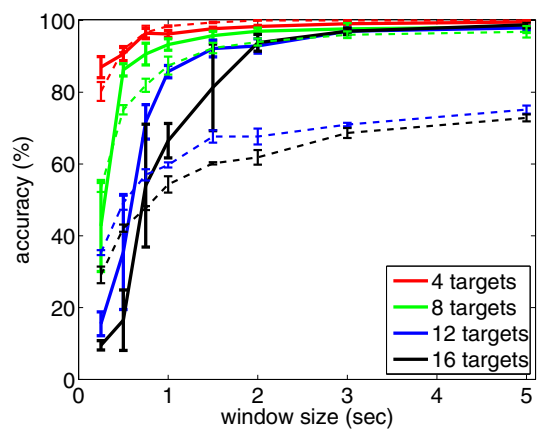

(b)

Fig. 2. (a) Decoding results averaged among all subjects. Stimulation frequency is $f=85 / 6 \mathrm{~Hz}$.(b) Decoding results for the same subjects as in Fig. 1c but for stimulation frequency $f=85 / 10=8.5 \mathrm{~Hz}$. Results are given for $4,8,12$, and 16 targets (phase shifts) and based on the MLMVN (solid lines) and extended for all electrodes method from [13] (dashed lines). Error bars show standard deviations.

8.5 Hz. As we can see from the result in Fig. 2b this choice of stimulus frequency improves the resulting accuracy. This suggests that the stimulation frequency is subject dependent and should be selected during a calibration stage.

Based on the results as presented above, we suggest the use of MLMVN in an on-line BCI, since in addition to the good accuracy, the training time is also reasonable.

In order to justify the use of MLMVN for phase-based SSVEP classification, we made a comparison with the classifiers used in the literature for such types of BCIs. The comparison with algorithms used in 14 is not straightforward, since they use only one EEG channel and consider only the principal oscillation harmonic. The generalization into several EEG channels requires some additional logic in the algorithm. But here we can stress, that since our approach allows (and involves) the use of a large amount of relevant information for decoding, we highly probably can achieve better performance. In addition, with the use of MLMVN, we do not need to apply additional logic for incorporating circularity of the data, since this is per se included into the classifier.

For comparison with [13, we have generalized the algorithm from this paper by taking into account all channels (instead of restricting ourselves to only the best one). This generalization has been done by taking the summation of the obtained correspondent projected values from all channels (compare to [13]). We believe, that such summation will not give worse results than using only one channel, since SSVEP responses are presented in all channels we have. To make a valid comparison, we used the same training set (with 4-fold cross-validation) and the same number of harmonics for decoding. Fig. [1]and 2 show the corresponding results in dashed lines. 
We notice that algorithms from [13] gives better results for small window sizes, but the obtained accuracy is not sufficient for reasonable use in BCI systems. For bigger window sizes MLMVN outperforms in the terms of accuracy. Inferior performance of MLMVN for small window sizes could be explained by the fact that here we do not have a clearly separable training set, which is tried to be fitted by such nonlinear classifier as MLMVN. Thus, while fitting badly separable training set, MLMVN is not able to make generalization (and we have a performance close to the chance level). While for more separable data (the case of bigger window size $T$ ), MLMVN is able to generalize data through much better nonlinear separation, then the classifier used in 13 . In addition, the comparison results suggest that for a small amount of target classes both classifiers produce almost equal results. And only starting from eight target classes MLMVN outperforms. The gain in accuracy for MLMVN becomes more prominent with the increase in number of classes.

For future steps in validation of MLMVN for use in a phase-based SSVEP BCI, different MLMVN topologies can be compared in order to increase the accuracy. Additionally to the phase information from each channel separately, we can also include features showing inter-channel relation, such as synchronization [20] and characteristics of propagating waves [21. We should also consider the construction of an on-line BCI system and its evaluation on larger subject group. For these purposes, a search for a proper subset of electrodes is also highly advisable (without significant loss in accuracy), as it increases the user-friendliness of the system.

\section{Conclusion}

We have performed decoding of stimuli-target information, encoded through phase shifts in SSVEP EEG data. The use of multilayer feedforward neural network based on multi-valued neurons (MLMVN) has been validated for this task, showing its superior performance for the bigger amount of target classes and the sufficient window size used in the decoding procedure. All these recommend the use of our approach for an on-line brain-computer interface.

Acknowledgments. NVM is supported by the Flemish Regional Ministry of Education (Belgium) (GOA 10/019). NC is supported by the European Commission (IST-2007-217077). AC and AR are supported by a specialization grant from the Agentschap voor Innovatie door Wetenschap en Technologie (IWT, Flemish Agency for Innovation through Science and Technology). MvV is supported by G.05809. MMVH is supported by research grants received from the Excellence Financing program (EF 2005) and the CREA Financing program (CREA/07/027) of the K.U.Leuven, the Belgian Fund for Scientific Research Flanders (G.0588.09), the Interuniversity Attraction Poles Programme - Belgian Science Policy (IUAP P6/054), the Flemish Regional Ministry of Education (Belgium) (GOA 10/019), and the European Commission (IST-2007-217077), and by the SWIFT prize of the King Baudouin Foundation of Belgium. 
The authors are also grateful to Refet Firat Yazicioglu, Tom Torfs and Cris Van Hoof from the Interuniversity Microelectronics Centre (imec) in Leuven for providing us the wireless EEG system.

\section{References}

1. Sajda, P., Müller, K.-R., Shenoy, K.V.: Brain-Computer Interfaces. IEEE Signal Proc. Magazine 25(1), 16-17 (2008)

2. Lebedev, M.A., Nicolelis, M.A.L.: Brain-Machine Interface: Past, Present and Future. Trends in Neurosc. 29(9), 536-546 (2005)

3. Manyakov, N.V., Van Hulle, M.M.: Decoding Grating Orientation from Microelectrode Array Recordings in Monkey Cortical Area V4. International Journal of Neural Systems 20(2), 95-108 (2010)

4. Velliste, M., Perel, S., Spalding, M.C., Whitford, A.S., Schwartz, A.B.: Cortical Control of a Prosthetic Arm for Self-Feeding. Nature 453, 1098-1101 (2008)

5. Birbaumer, N., Kübler, A., Ghanayim, N., Hinterberger, T., Perelmouter, J., Kaiser, J., Iversen, I., Kotchoubey, B., Neumann, N., Flor, H.: The Thought Translation Device (TTD) for Completely Paralyzed Patients. IEEE Transactions on Rehabilitation Egineering 8(2), 190-193 (2000)

6. Blankertz, B., Dornhege, G., Krauledat, M., Müller, K.-R., Curio, G.: The NonInvasive Berlin Brain-Computer Interface: Fast Acquisition of Effective Performance in Untrained Subjects. Neuroimage 37(2), 539-550 (2007)

7. Herrmann, C.S.: Human EEG Responses to 1-100 Hz Flicker: Resonance Phenomena in Visual Cortex and Their Potential Correlation to Cognitive Phenomena. Exp. Brain Res. 137, 346-353 (2001)

8. Cheng, M., Gao, X., Gao, S., Xu, D.: Design and Implementation of a BrainComputer Interface with High Transfer Rates. IEEE Transactions on Biomedical Engineering 49(10), 1181-1186 (2002)

9. Gao, Y., Wang, R., Gao, X., Hong, B., Gao, S.: A Practical VEP-based BrainComputer Interface. IEEE Transactions on Neural Systems and Rehabilitation Engineering 14(2), 234-240 (2006)

10. Manyakov, N.V., Chumerin, N., Combaz, A., Robben, A., Van Hulle, M.M.: Decoding SSVEP Responses Using Time Domain Classification. In: Proc. of the International Conference on Fuzzy Computation and 2nd International Conference on Neural Computation, pp. 376-380 (2010)

11. Allison, B., Luth, T., Valbuena, D., Teymourian, A., Volosyak, I., Graser, A.: BCI Demographics: How Many (and What Kinds of) People Can Use an SSVEP BCI? IEEE Transactions on Neural Systems and Rehabilitation Engineering 18(2), 107$116(2010)$

12. Volosyak, I., Cecotti, H., Gräser, A.: Impact of Frequency Selection on LCD Screens for SSVEP Based Brain-Computer Interfaces. In: Cabestany, J., Sandoval, F., Prieto, A., Corchado, J.M. (eds.) IWANN 2009, Part I. LNCS, vol. 5517, pp. 706-713. Springer, Heidelberg (2009)

13. Jia, C., Gao, X., Hong, B., Gao, S.: Frequency and Phase Mixed Coding in SSVEP-based Brain-Computer Interface. IEEE Transaction on Biomedical Engineering 58(1), 200-206 (2011)

14. Lee, P.-L., Sie, J.-J., Liu, Y.-J., Wu, C.-H., Lee, M.-H., Shu, C.-H., Li, P.-H., Sun, C.-W., Shyu, K.-K.: An SSVEP-Actuated Brain Computer Interface Using Phase-Tagged Flickering Sequences: A Cursor System. Annals of Biomedical Engineering 38(7), 2383-2397 (2010) 
15. Aizenberg, I., Moraga, C.: Multilayer Feedforward Neural Network Based on Multivalued Neurons (MLMVN) and a Backpropagation Learning Algorithm. Soft Comput. 11, 169-183 (2007)

16. Yazicioglu, R.F., Torfs, T., Merken, P., Penders, J., Leonov, V., Puers, R., Gyselinckx, B., Van Hoof, C.: Ultra-low-power biopotential interfaces and their applications in wearable and implantable systems. Microel. J. 40(9), 1313-1321 (2009)

17. Aizenberg, I., Paliy, D., Zurada, J.M., Astola, J.: Blur Identification by Multilayer Neural Network based on Multi-Valued Neurons. IEEE Transactions on Neural Networks 19(5), 883-898 (2008)

18. Aizenberg, I.: Complex-Valued Neurons with Phase-Dependent Activation Functions. In: Rutkowski, L., Scherer, R., Tadeusiewicz, R., Zadeh, L.A., Zurada, J.M. (eds.) ICAISC 2010, Part. II. LNCS (LNAI), vol. 6114, pp. 3-10. Springer, Heidelberg (2010)

19. Strasburger, H.: The Analysis of Steady State Evoked Potentials Revised. Clin. Vis. Sci. 1(3), 245-256 (1987)

20. Manyakov, N.V., Van Hulle, M.M.: Synchronization in Monkey Visual Cortex Analyzed with an Information-Theoretic Measure. Chaos 18(3), 037130 (2008)

21. Manyakov, N.V., Vogels, R., Van Hulle, M.M.: Decoding Stimulus-Reward Pairing from Local Field Potentials Recorded from Monkey Visual Cortex. IEEE Transactions on Neural Networks 21(12), 1892-1902 (2010) 